Check for updates

Cite this: Mater. Adv., 2021, 2, 4093

Received 30th March 2021 Accepted 12th May 2021

DOI: 10.1039/d1ma00284h

rsc.li/materials-advances

\title{
A reduced graphene oxide/bismuth vanadate composite as an efficient piezocatalyst for degradation of organic dye
}

\author{
Manish Kumar, $\dagger$ Gurpreet Singh (D) $\dagger$ and Rahul Vaish (DD *
}

\begin{abstract}
Reduced graphene oxide $(\mathrm{rGO}) /$ bismuth vanadate $\left(\mathrm{BiVO}_{4}\right)$ composites with varying $\mathrm{rGO}$ content $(0,1,2$, 3 , and $5 \mathrm{wt} \%$ ) were synthesized and explored for their piezocatalytic dye degradation performance. $\mathrm{X}$-Ray diffraction (XRD) and Raman spectroscopy evidenced the co-existence of $\mathrm{BiVO}_{4}$ and $\mathrm{rGO}$ phases in all composite samples. The interfacial coupling between the $\mathrm{BiVO}_{4}$ and $\mathrm{rGO}$ phases in a typical $\mathrm{rGO} / \mathrm{BiVO}_{4}$ composite was confirmed using transmission electron microscopy (TEM). The elemental

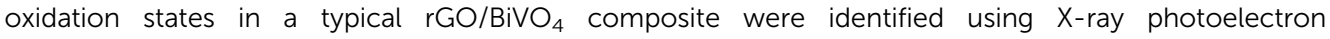
spectroscopy (XPS). The piezocatalytic activity of all samples was analyzed by the use of a model dye named methylene blue (MB) using an ultrasonicator. Results demonstrate that the piezocatalytic activity greatly depends on the rGO content of the composite. The piezocatalytic dye degradation performance of the composites showed an initial increase with increment in rGO content up to $2 \mathrm{wt} \%$ followed by a decrease with further increment in rGO content. The best piezocatalytically active composite sample ( 2 wt $\% \mathrm{rGO} / \mathrm{BiVO}_{4}$ ) showed $\sim 81 \% \mathrm{MB}$ dye degradation as compared to pure $\mathrm{BiVO}_{4}(\sim 19 \%)$ under $180 \mathrm{~min}$ ultrasonication. Thus, the $2 \mathrm{wt} \% \mathrm{rGO} / \mathrm{BiVO}_{4}$ composite has promising piezocatalytic capability to be used for water remediation.
\end{abstract}

\section{Introduction}

The water pollution crisis is a prevailing concern these days due to the rapid development of mankind and industry. ${ }^{1}$ The discharge of colored organic dyes from the textile industry into natural water sources is one of the main sources causing water pollution. $^{2}$ This problem has attracted the attention of many researchers. Recently, the piezocatalysis process has emerged as a promising technology for water remediation, which has been related to piezoelectric materials. ${ }^{3}$ The presence of a polarization field inside piezoelectric materials under stimulation with mechanical strain retards recombination of free charge carriers (electrons, $\mathrm{e}^{-}$; holes, $\mathrm{h}^{+}$). ${ }^{3}$ These generated free charge carriers further participate in the surface redox reactions generating reactive oxygen species (ROS) such as ${ }^{\bullet} \mathrm{OH}$ and ${ }^{\bullet} \mathrm{O}_{2}{ }^{-}$. The ROS further decompose dye molecules into simpler products. $^{4-6}$ Piezocatalysis has been mainly reported for organic pollutant degradation. ${ }^{7} \mathrm{ZnO},{ }^{8} \mathrm{MoS}_{2},{ }^{9} \mathrm{NaNbO}_{3},{ }^{10} \mathrm{PbTiO}_{3},{ }^{11}$ $\mathrm{BaTiO}_{3},{ }^{12}$ etc., have already been reported for piezocatalytic applications. In the piezocatalysis field, much attention has

School of Engineering, Indian Institute of Technology Mandi, Mandi, Himachal Pradesh 175005, India. E-mail: rahul@iitmandi.ac.in

$\dagger$ Both authors have equally contributed. been given to piezocatalysis using $\mathrm{BaTiO}_{3}$ catalyst. $\mathrm{BaTiO}_{3}$ crystals in the form of nanowires, nano-dendrites, and nanoparticles exhibited promising piezocatalytic dye degradation ${ }^{13,14}$ and water-splitting. ${ }^{14}$ Various composites/heterostructures of $\mathrm{BaTiO}_{3}$ such as $\mathrm{BiOX} / \mathrm{BaTiO}_{3}\left(\mathrm{X}=\mathrm{Cl}, \mathrm{Br}\right.$, and $\left.\mathrm{Cl}_{0.166} \mathrm{Br}_{0.834}\right),{ }^{13} \mathrm{BaTiO}_{3} /$ graphene, ${ }^{15}$ and iodide-doped $\mathrm{BiVO}_{4} / \mathrm{BaTiO}_{3}{ }^{16}$ have also been reported to show superior piezocatalysis over bare $\mathrm{BaTiO}_{3}$. The dye degradation can further be enhanced using combined photopiezocatalysis. In this direction, Zhou et al. achieved 97\% RB dye degradation within 30 min during combined photo-piezocatalysis using $\mathrm{ZnO} / \mathrm{BaTiO}_{3}$ heterostructures, which was higher than dye degradation achieved using only a light source and only sonication. ${ }^{17}$ However, this new field still needs identification and exploration of new piezocatalytically active materials with promising performance. ${ }^{6,18}$

Monoclinic bismuth vanadate $\left(\mathrm{m}-\mathrm{BiVO}_{4}\right)$ exhibits excellent technological properties such as solar energy absorption, ionic conductivity, ferroelasticity, less toxicity, coloristic properties, ${ }^{19}$ eco-friendliness, and high chemical stability, and is costeffective. $^{20,21}$ It has been widely reported for its photocatalytic application under visible light stimulation. Though $\mathrm{BiVO}_{4}$ has a centro-symmetric structure, it has been reported to be piezoresponsive due to the flexoelectric effect. ${ }^{22}$ Recently, in our previously published work, $\mathrm{BiVO}_{4}$ ceramic pellets demonstrated enhanced piezocatalytic dye degradation capability in addition 
to photocatalytic activity. ${ }^{23}$ However, the piezocatalytic performance of $\mathrm{BiVO}_{4}$ should be further improved.

Reduced graphene oxide (rGO) has a 2-D conjugated structure with a very high surface area along with high electrical conductivity and carrier mobility. ${ }^{24}$ The composite formed between $\mathrm{BiVO}_{4}$ and rGO showed enhanced photocatalytic activity compared with pristine $\mathrm{BiVO}_{4}$ through enhancement of free charge carrier separation. ${ }^{25,26}$ Likewise, it is expected that the $\mathrm{rGO} /$ $\mathrm{BiVO}_{4}$ composite will also exhibit enhanced piezocatalytic performance. So, in this present study, $\mathrm{rGO} / \mathrm{BiVO}_{4}$ composites (varying in rGO content) are investigated for their piezocatalytic performance.

\section{Materials and methods}

\subsection{Synthesis of BiVO4 ceramic powder}

Firstly, $\mathrm{BiVO}_{4}$ ceramic powder was synthesized using $\mathrm{Bi}_{2} \mathrm{O}_{3}$ and $\mathrm{V}_{2} \mathrm{O}_{5}$ oxides as starting powders, taken in accordance with their stoichiometric molar ratios, which were manually mixed and ground for about $30 \mathrm{~min}$. The procured homogenous and finely powdered mixture was subjected to calcination at $700{ }^{\circ} \mathrm{C}$ for a duration of $8 \mathrm{~h}$ for the formation of the $\mathrm{BiVO}_{4}$ phase. The obtained $\mathrm{BiVO}_{4}$ powder was ball milled for 12 hours at $250 \mathrm{rpm}$.

\subsection{Synthesis of $\mathrm{rGO} / \mathrm{BiVO} 4$ composites}

To synthesize $\mathrm{rGO} / \mathrm{BiVO}_{4}$ composites, the calculated amounts (1 wt $\%, 2 \mathrm{wt} \%, 3 \mathrm{wt} \%$, and $5 \mathrm{wt} \%$ ) of $\mathrm{rGO}$ were initially dispersed in $25 \mathrm{ml}$ ethanol solution in separate vials followed by adding the remaining $\mathrm{wt} \%$ of $\mathrm{BiVO}_{4}$ sample to each of them. Further, ultrasonication was carried out for $45 \mathrm{~min}$ at $30{ }^{\circ} \mathrm{C}$ until a homogenous solution was obtained. The solution was then irradiated using 3 Havells LED bulbs with $15 \mathrm{~W}$ power each for a duration of $48 \mathrm{~h}$ with stirring in order to achieve more reduction of $\mathrm{rGO}$ using $\mathrm{BiVO}_{4}$ photocatalytic activity. The procured sample was transferred to a $100 \mathrm{ml}$ capacity, Teflon lined stainless steel autoclave for hydrothermal treatment for forming a better connection between $\mathrm{rGO}$ and $\mathrm{BiVO}_{4}$. After filling $40 \%$ volume with solution, the autoclave was sealed and kept at a temperature of $200{ }^{\circ} \mathrm{C}$ for $8 \mathrm{~h}$ and then further cooled to room temperature. The precipitates procured were washed thoroughly with ethanol and distilled water 3 times. The obtained products were dried in an oven at $70{ }^{\circ} \mathrm{C}$ for 4 hours. The total synthesis process is schematically shown in Scheme 1.

\subsection{Characterization}

The structural phases of all the synthesized samples were identified through an X-ray diffractometer (XRD) using a Rigaku diffractometer, Japan (9 kW Cu-K $\alpha$ anode). Sample scan was carried out over the scan range of 10-90 with a scan rate of $3^{\circ} \mathrm{min}^{-1}$. Raman spectroscopy was used to find the bonding and structure that exist in all samples. Raman spectra were acquired using a LabRAM HR Evolution Raman spectrometer (HORIBA, Japan) where 10\% power and $532 \mathrm{~nm}$ laser excitation wavelength along with 600 gratings were used to scan the samples. A scan range of $100-2000 \mathrm{~cm}^{-1}$ was provided. A Nova
Nano SEM-450 (JFEI, USA) field emission scanning electron microscope (FE-SEM) was used to analyze the microstructures and morphologies of the samples. A UV-visible spectrophotometer (Shimadzu-2600) was used for recording the absorbance spectra of the samples. The interfacial coupling between rGO and $\mathrm{BiVO}_{4}$ in one composition was confirmed through a transmission electron microscope (TEM) with the use of an FP-Tecnai G2 S-TWIN TEM instrument (FEI, USA). To analyze the compositional chemical states of elements, X-ray photoelectron spectroscopy (XPS) was used. A Nexsa X-ray photoelectron spectrophotometer with an Al-K $\alpha$ source was used to obtain XPS spectra.

\subsection{Piezocatalytic experiments}

The piezocatalytic performances of all the $\mathrm{rGO} / \mathrm{BiVO}_{4}$ samples were analyzed using piezocatalytic experiment under ultrasonication. $0.05 \mathrm{~g}$ of each sample was utilized. Before starting the piezocatalytic experiment, complete adsorption of all the samples in connection with the dye solution was achieved in the dark with continuous stirring at the rate of $500 \mathrm{rpm}$. Once adsorption saturation was achieved, the previously used dye was replaced with fresh $10 \mathrm{ml}$ dye (initial dye concentration $\sim 5 \mathrm{mg} \mathrm{l}^{-1}$ ). The dye solution along with the sample in it was subjected to ultra-sonication. For providing the ultrasonic vibrations, an ultrasonicator with $70 \mathrm{~W}$ power and $40 \mathrm{kHz}$ frequency with water acting as a medium was used. Absorbance spectral measurement was done with $1 \mathrm{ml}$ of MB dye solution after every $30 \mathrm{~min}$ of ultrasonication and the constant volume was maintained by recovering the dye to the beaker. Water was used as an ultrasonication medium and was replaced after at most 15 min duration to avoid the dye solution heating. To determine the percentage removal of $\mathrm{MB}$ dye from aqueous solution, the formula in eqn (1) was used. ${ }^{27}$

$$
\% \text { removal of } \mathrm{MB} \text { dye }=\frac{C_{0}-C}{C_{0}} \times 100
$$

where the symbols $C_{0}$ and $C$ denote the MB dye solution concentration initially and at a time ' $t$ ' respectively.

\section{Results and discussion}

Fig. 1 shows XRD patterns of BV, rGO, 1rG-BV, 2rG-BV, 3rG$\mathrm{BV}$, and $5 \mathrm{rG}-\mathrm{BV}$ powders. The obtained sharp XRD peaks in the pure BV and all rG-BV samples indicated high crystallinity. All XRD peaks in the BV sample showed a good match with peaks reported in standard JCPDS 01-075-1866 for a pure monoclinic phase of $\mathrm{BiVO}_{4}$. The peaks of the $\mathrm{rG}-\mathrm{BV}$ composites are similar to that of $\mathrm{BV}$ except for an extra peak at $\sim 27.2^{\circ}$, confirming the monoclinic phase of $\mathrm{BiVO}_{4}$ in all rG-BV composites. ${ }^{28}$ The extra peak at $\sim 27.2^{\circ}$ is associated with the (002) plane of the rGO phase. Generally, the (002) plane of graphite showed a peak at $26.5{ }^{\circ}{ }^{29} \mathrm{In}$ most of the literature, the (002) peak of rGO has been reported at a $2 \theta$ angle which is less than that found in the case of graphite $\left(26.5^{\circ}\right)$. But in the present case, the rGO peak was found at the same $2 \theta$ angle of $26.5^{\circ}$. The peak of rGO at $26.5^{\circ}$ is similar to that of graphite, which is due to the restacking of the 

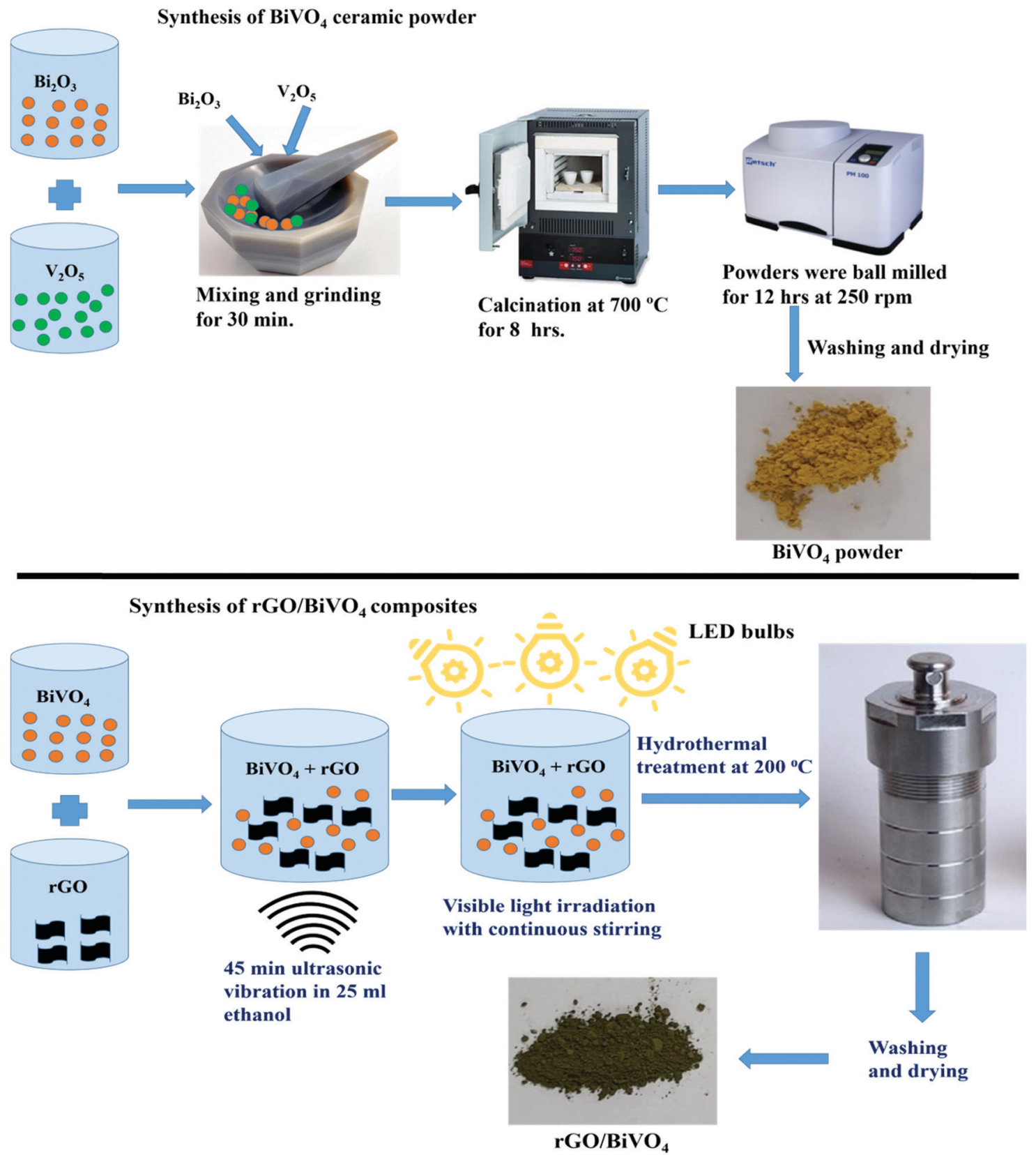

Scheme 1 Synthesis process of $r G-B V$ composites.

rGO layers. There is slight shift in the peaks from $26.5^{\circ}$ to $27.2^{\circ}$ in the $\mathrm{rG}-\mathrm{BV}$ composites, which may be due to the enhanced restacking of rGO layers and/or further reduction of rGO during synthesis through ultrasonication, photocatalytically induced reduction and hydrothermal treatment. ${ }^{29-31}$ Thus, XRD peaks affirm the presence of both $\mathrm{rGO}$ and $\mathrm{BiVO}_{4}$ in the prepared composites with high crystallinity. The as-prepared powdered samples are shown along with their respective XRD patterns.

Raman spectra of BV and all rG-BV composites are shown in Fig. 2. Raman bands at around 118, 204, 324, 360, and $818 \mathrm{~cm}^{-1}$ in both $\mathrm{BV}$ and all rG-BV composites correspond to the $\mathrm{BiVO}_{4}$ phase. ${ }^{32}$ The strongest band at $818 \mathrm{~cm}^{-1}$ is due to the symmetric stretching of $\mathrm{V}-\mathrm{O}$ in the crystal structure. Two other
Raman modes at 324 and $360 \mathrm{~cm}^{-1}$ were viewed because of the symmetric and antisymmetric bending of $\mathrm{V}-\mathrm{O}$ bonds, respectively. ${ }^{33}$ The observed external modes at 118 and $204 \mathrm{~cm}^{-1}$ were because of the translation and rotation of the $\mathrm{BiVO}_{4}$ crystal lattice structural units. In addition to these bands, two additional Raman bands at 1356 and $1594 \mathrm{~cm}^{-1}$ were viewed in the case of all rG-BV composites, which were associated with rGO carbon, which have been claimed in the literature as $\mathrm{G}$ and $\mathrm{D}$ bands, respectively. ${ }^{19}$ The $\mathrm{G}$ band represents the in-planar stretching of symmetric $\mathrm{sp}^{2} \mathrm{C}-\mathrm{C}$ bonds, while the $\mathrm{D}$ band represents the interruption that occurred in the symmetric hexagonal graphitic lattice. ${ }^{34}$ Additionally, there was a little shift observed in the D and G bands of the 1rG-BV, 2rG-BV, 


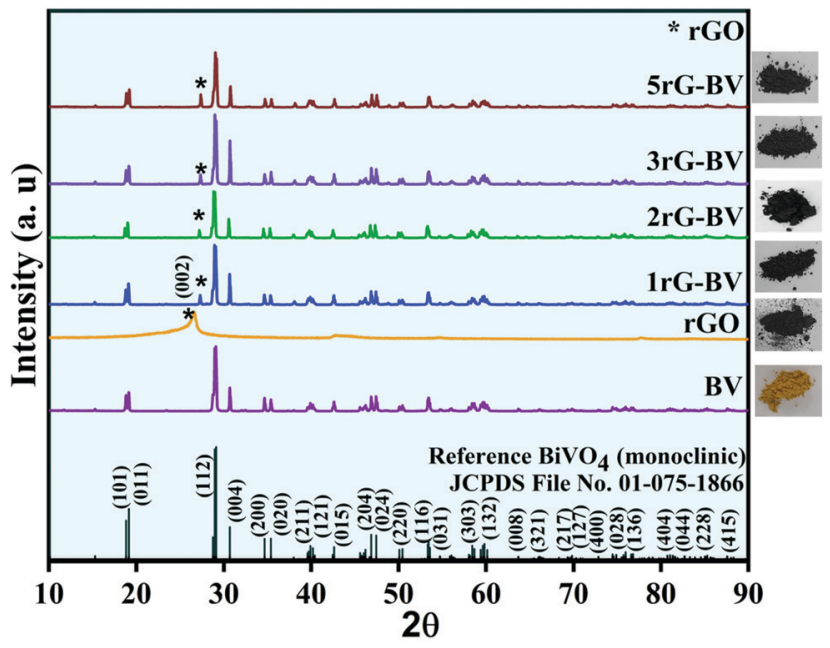

Fig. 1 XRD patterns of $B V, r G O, 1 r G-B V, 2 r G-B V, 3 r G-B V$, and $5 r G-B V$ powders.

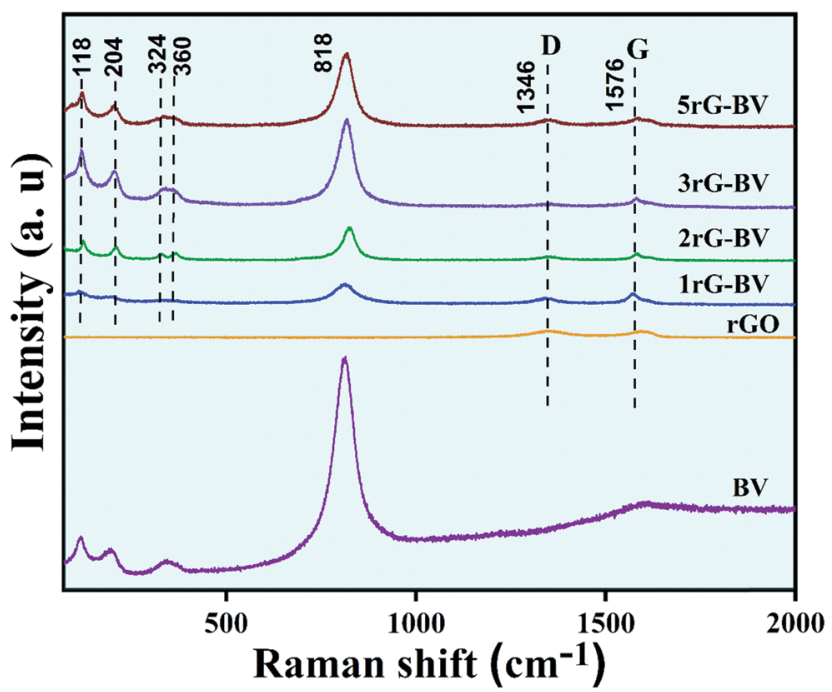

Fig. 2 Raman spectra of BV, rGo, 1rG-BV, 2rG-BV, 3rG-BV, and $5 \mathrm{rG}-\mathrm{BV}$.

3rG-BV, and 5rG-BV samples as compared to rGO, which might have resulted from the changed surface strain during the interaction of $\mathrm{rGO}$ with $\mathrm{BiVO}_{4}$ in the composites. ${ }^{35}$

SEM micrographs showing the morphologies of $\mathrm{BV}$, rGO, 1rG-BV, and 5rG-BV are presented in Fig. 3(a-d). In the BV sample, the irregular morphology of $\mathrm{BiVO}_{4}$ particles was visualized as shown in Fig. 3(a). The rGO layered structure can be viewed in Fig. 3(b). The accumulation of rGO layers on $\mathrm{BiVO}_{4}$ particles in the case of $2 \mathrm{rG}-\mathrm{BV}$ and $5 \mathrm{rG}-\mathrm{BV}$ can be seen in Fig. 3(c and d), which reveals an adequate contact and interfacial interaction between the $\mathrm{BiVO}_{4}$ and $\mathrm{rGO}$ phases.

TEM micrographs of a typical composite sample (2rG-BV) are shown in Fig. 4(a and b). Fig. 4(a) shows the presence of rGO layers and $\mathrm{BiVO}_{4}$ crystals in the 2rG-BV composite. Fig. 4(b) shows the well-defined and tight interfacial coupling formed between the rGO and $\mathrm{BiVO}_{4}$ phases in the 2rG-BV composite.

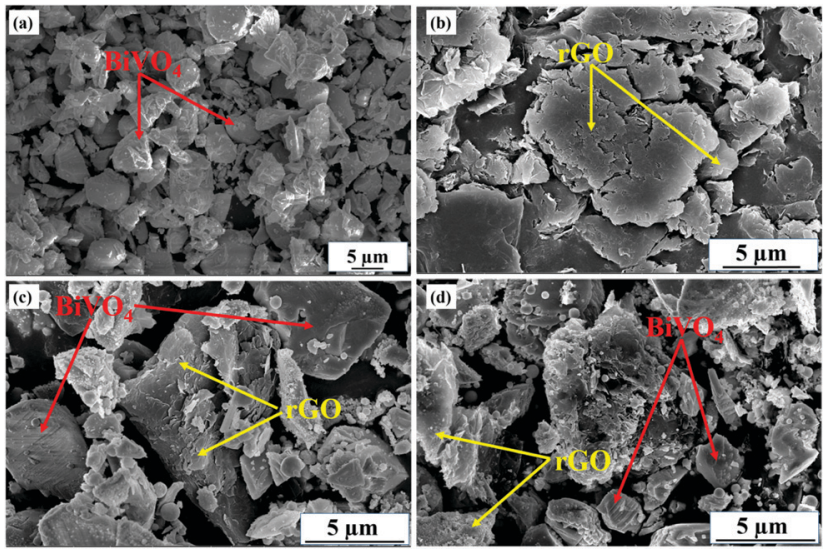

Fig. 3 SEM micrographs of (a) rGO, (b) BV, (c) 2rG-BV and (d) 5rG-BV.

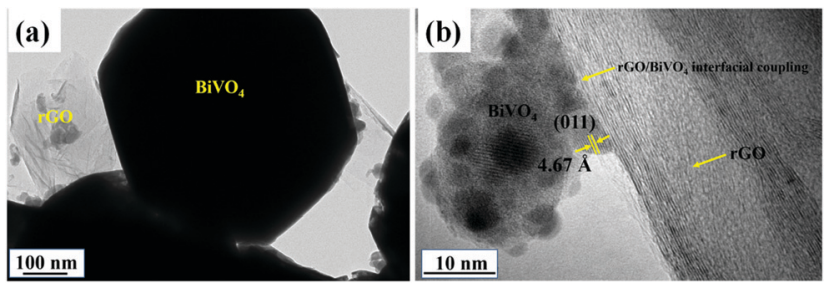

Fig. 4 ( $a$ and b) TEM micrographs of $2 r G-B V$ showing the formation of interfacial coupling between $\mathrm{rGO}$ and $\mathrm{BiVO}_{4}$

The observed fringe spacing $d=4.67 \AA$ corresponds to the $\mathrm{BiVO}_{4}(011)$ crystallographic plane. ${ }^{23}$ The right side shows multiple rGO layers. Thus, the TEM evidenced the existence of appropriate interfacial coupling between the rGO and $\mathrm{BiVO}_{4}$ phases in the rG-BV composites. This interface formed will support the transport of electrons from $\mathrm{BiVO}_{4}$ to the rGO phase and thus will assist in effective charge separation within the composite during the dye degradation.

The XPS spectra of a typical composite sample (2rG-BV) showing V2p, Bi4f, C1s, and O1s scans are presented in Fig. 5(a-d). In the case of the Bi4f spectrum, the peaks displayed $\mathrm{Bi}_{4 / 2}$ and $\mathrm{Bi} 4 \mathrm{f}_{5 / 2}$ components which further deconvoluted into the components $\mathrm{Bi}^{3+}$ and $\mathrm{Bi}^{2+} \cdot{ }^{36}$ The presence of the $\mathrm{Bi}^{3+}$ oxidation state in the sample can be confirmed by the high peaks positioned at 159.2 and $165 \mathrm{eV}$, while the weaker peaks at 157 and $163 \mathrm{eV}$ confirm the $\mathrm{Bi}^{2+}$ oxidation state in the sample. For the V2p scan, the split peaks displayed $V 2 \mathrm{p}_{1 / 2}$ and $\mathrm{V} 2 \mathrm{p}_{3 / 2}$ peaks which further deconvoluted into components $\mathrm{V}^{4+}$ and $\mathrm{V}^{5+} \cdot{ }^{37}$ The presence of the $\mathrm{V}^{5+}$ oxidation state in the sample can be confirmed by the peaks positioned at 159.2 and $165 \mathrm{eV}$, while the high peaks at 157 and $163 \mathrm{eV}$ confirm the existence of some $\mathrm{V}^{4+}$ oxidation state in the sample. In the O1s scan spectrum, there exists an asymmetric component O1s which is further deconvoluted into $\mathrm{O}_{\mathrm{L}}$ and $\mathrm{O}_{\mathrm{A}}$ components. The $\mathrm{O}_{\mathrm{L}}$ component positioned at $530.01 \mathrm{eV}$ corresponds to the lattice oxygen $\left(\mathrm{O}^{2-}\right)$, while the $\mathrm{O}_{\mathrm{A}}$ component positioned at $531.20 \mathrm{eV}$ corresponds to oxygen vacancies along with many other adsorbed oxygen species. ${ }^{36,38}$ The intrinsic defects formed 

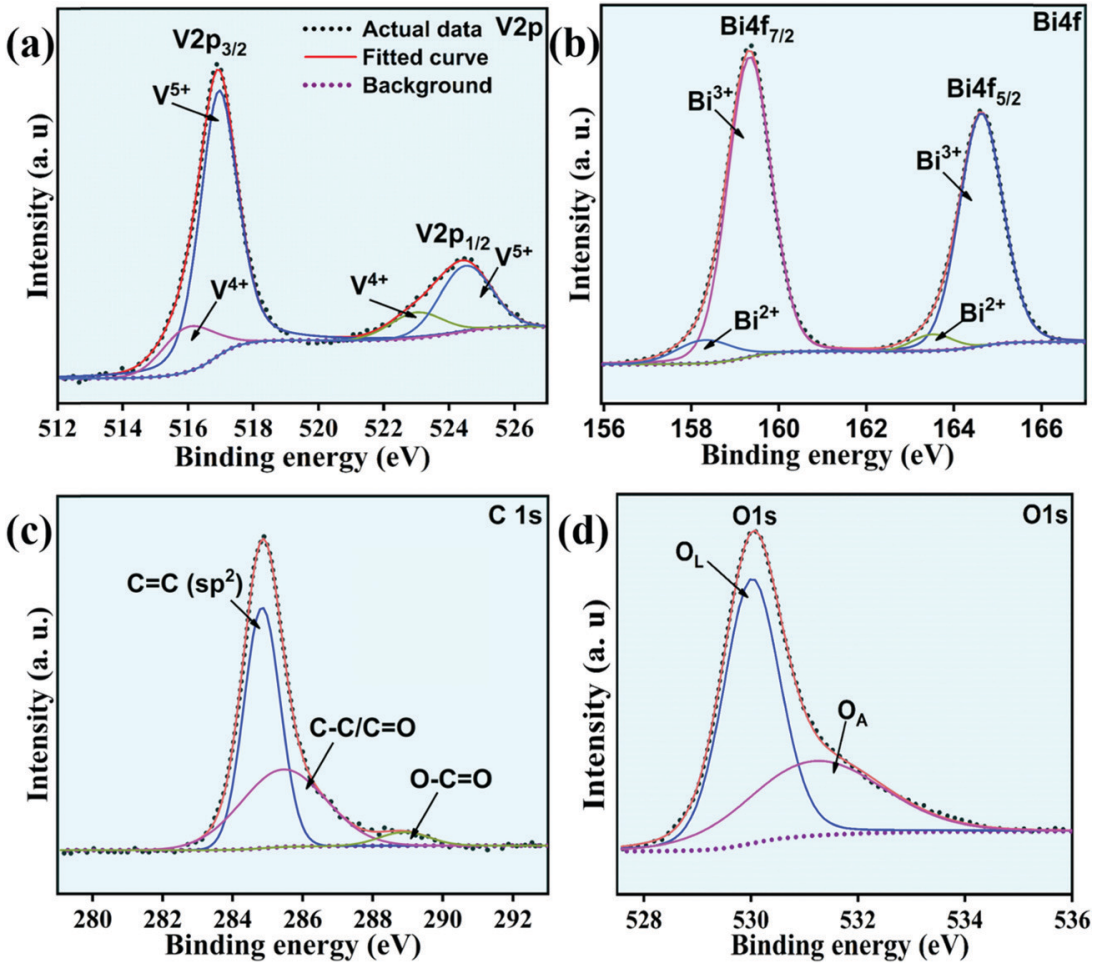

Fig. 5 The XPS spectra of the 2rG-BV sample: (a) V2p, (b) Bi4f, (c) C1s and (d) O1s.

during thermal treatment (i.e., localized oxygen vacancies) trap the charge in excess as electron pairs which results in the reduction of $\mathrm{V}^{5+}$ to $\mathrm{V}^{4+}$ and $\mathrm{Bi}^{3+}$ to $\mathrm{Bi}^{2+}$. This leads to the appearance of signal peaks of $\mathrm{Bi}^{2+}$ and $\mathrm{V}^{4+}$ along with those of $\mathrm{Bi}^{3+}$ and $\mathrm{V}^{5+}$ in the XPS spectra. For rGO confirmation in the 2rG-BV sample, C species can be detected. ${ }^{39}$ For this, C1s scan was performed and deconvoluted into 3 peaks as shown in Fig. 5(c). The deconvoluted peaks at $\sim 284.6 \mathrm{eV}, \sim 286.4$ and $\sim 288.6 \mathrm{eV}$ indicate the $\mathrm{sp}^{2}$ hybridized carbon (C-C) species, oxygen-containing functional group (C-O, epoxy, and hydroxyl) species and $\left(\mathrm{C}=\mathrm{C}\right.$, carboxyl) species, respectively. ${ }^{25}$

To investigate the piezocatalytic performance of the rGO$\mathrm{BiVO}_{4}$ composite samples, piezocatalytic degradation of MB dye was done, and the acquired results are shown in Fig. 6(a-c). It is to be taken into account that here adsorption saturation was achieved cautiously prior to the commencement of the piezocatalytic test using an ultrasonicator. The concentration of $\mathrm{MB}$ dye during the piezocatalytic experiment was calculated by recording an absorbance peak at $664 \mathrm{~nm}$. The absorbance spectra obtained during the piezocatalytic experiment with the use of the 2rG-BV composite are displayed in Fig. 6(a). The absorbance peak intensity was observed to continuously decrease with vibration time, which indicates a decrease in MB dye concentration in the dye solution. Fig. 6(b) displays the $\frac{C}{C_{0}}$ vs. vibration time plots for piezocatalytic experiments without
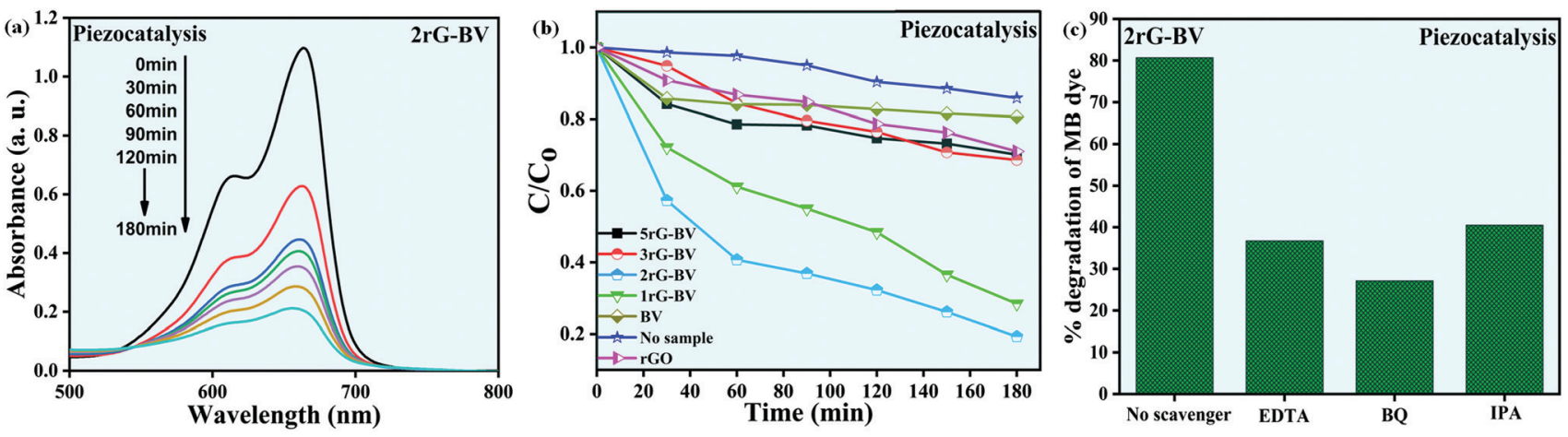

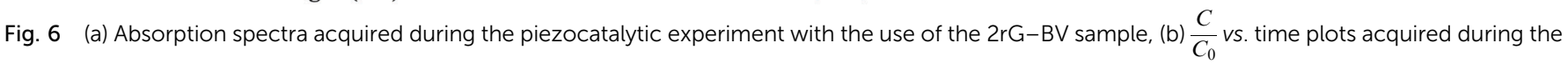

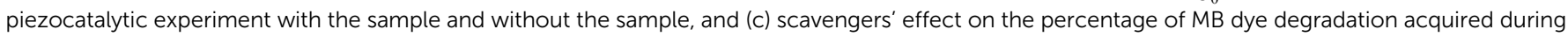
the piezocatalytic experiment with the use of the $2 r G-B V$ sample. 
and with the use of the samples. Under ultrasonication, only $\sim 15 \%$ dye degradation was obtained with no sample. During the ultrasonication process, the constant formation, growth, and collapse of water bubbles take place, which gives rise to the formation of local hot spots with the localized temperature commencing near $4000-5000 \mathrm{~K}^{40}$ Since there is an occurrence of localized hot spots, ${ }^{\bullet} \mathrm{OH}$ radicals are formed due to the thermolytic decomposition of water. The generated ${ }^{\bullet} \mathrm{OH}$ radicals are further used for MB dye degradation. ${ }^{40}$ This overall process has been referred to as sonolysis in the literature. ${ }^{41}$ With the use of the BV, rGO, 1rG-BV, 2rG-BV, 3rG-BV, and 5rG-BV samples, the dye degradation achieved was $19 \%, 29 \%, 72 \%, 81 \%, 31 \%$, and $30 \%$, respectively, under $180 \mathrm{~min}$ of ultrasonication. The results show that the piezocatalytic activity greatly depends on the rGO content of the composite. The piezocatalytic dye degradation performance of the composites showed an initial increase with increment in rGO content up to $2 \mathrm{wt} \%$ followed by a decrease with further increment in rGO content. The best piezocatalytically active composite sample ( $2 \mathrm{wt} \% \mathrm{rGO} / \mathrm{BiVO}_{4}$ ) showed $\sim 81 \% \mathrm{MB}$ dye degradation as compared to pure $\mathrm{BiVO}_{4}(\sim 19 \%)$ under $180 \mathrm{~min}$ ultrasonication. Scavengers such as $p$-benzoquinone ( $p$-BQ), ethylenediaminetetraacetic acid (EDTA), and isopropanol (IPA) were added separately into the dye solution during piezocatalytic experiments in view of trapping active species like superoxide radicals $\left({ }^{\bullet} \mathrm{O}_{2}{ }^{-}\right)$, holes $\left(\mathrm{h}^{+}\right)$, and hydroxyl radicals $\left({ }^{\bullet} \mathrm{OH}\right)$ respectively. With the capture of the main active species there occurs a reduction of piezocatalytic performance. ${ }^{4,42,43}$ Fig. 6(c) clearly shows that the BQ scavenger drastically has an impact on the piezocatalytic activity, which scavenges superoxide $\left({ }^{\bullet} \mathrm{O}_{2}{ }^{-}\right)$ radicals. This specifies that ${ }^{\bullet} \mathrm{O}_{2}{ }^{-}$radicals acted as the main active radical species behind piezocatalytic dye degradation. ${ }^{44}$ The 2rG-BV sample showed the best piezocatalytic activity among all the synthesized samples. This might be due to the fact that at higher wt $\%$ of reduced graphene oxide, most of the surface of $\mathrm{BiVO}_{4}$ gets covered with rGO layers, which reduces the piezocatalytic performance. 2rG-BV is an optimum composite for the best piezocatalytic performance under study.

A proposed piezocatalytic reaction mechanism for the $\mathrm{BiVO}_{4}, \mathrm{rGO}$, and $\mathrm{rGO} / \mathrm{BiVO}_{4}$ composite for $\mathrm{MB}$ dye degradation is schematically shown in Fig. 7. As per the literature, $\mathrm{BiVO}_{4}$ is known to possess localized surface piezoelectricity. ${ }^{22}$ Due to the localized surface piezoelectricity, polarization charges (internal electric field) are induced in $\mathrm{BiVO}_{4}$, when mechanical stress in the form of ultrasonication vibration is provided. ${ }^{45}$ The free electrons and holes within $\mathrm{BiVO}_{4}$ get attracted, and move in opposite directions towards the opposite polarity of the internal electric field. In this way, the recombination of free electrons and holes is inhibited, which results in more transportation of these free charge carriers to the $\mathrm{BiVO}_{4}$ surface, ${ }^{46-48}$ which further participate in piezocatalytic surface reactions. Moreover, the piezoelectric polarization field also provides further band bending, which further assists in the easy transfer of free carriers to adsorbed pollutants undergoing piezocatalytic reactions. ${ }^{4,46,49}$ It is suggested in many studies that free charge carriers inside piezoelectric materials are due to the existence of defects in them at room temperature. ${ }^{4,50}$ Also, the temperature

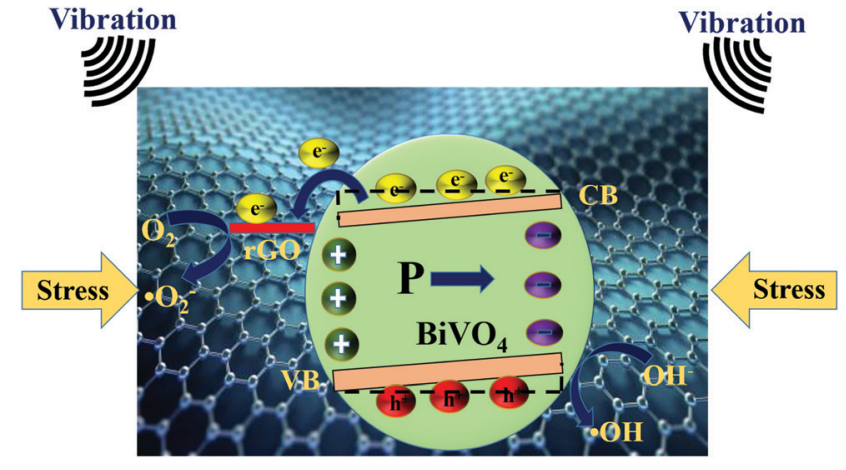

Fig. 7 Piezocatalytic reaction mechanism for the rG-BV composites.

rise due to the hot spots results in thermal emission of electrons from the valence band to the conduction band of the semiconductor. ${ }^{50,51}$ In the present work, rGO also shows some amount of dye degradation. It has been suggested in the literature that the rGO edges contain unsaturated carbon and other defects present on rGO may serve as catalytically active centers for catalytic activity. ${ }^{52}$ Moreover, it has been also suggested in the literature that the adsorption of organic dye also increases under ultrasonication, which may have some contribution here. ${ }^{53}$ In case of the $\mathrm{rGO} / \mathrm{BiVO}_{4}$ composite, the mechanism is slightly different. In fact in this case, the free electrons in the $\mathrm{BiVO}_{4}$ surface readily transfer from $\mathrm{BiVO}_{4}$ to rGO due to multiple favorable conditions. Bai et al. reported that when n-type $\mathrm{BiVO}_{4}$ and rGO come into contact with each other through the formation of a heterojunction/composite, the flow of electrons from $\mathrm{BiVO}_{4}$ to rGO takes place until the Fermi level of the system reaches a balance (otherwise before contact, the Fermi level of rGO is higher than that of $\mathrm{BiVO}_{4}$ and the work function of rGO $(4.75 \mathrm{eV})$ is smaller than that of $\mathrm{BiVO}_{4}$ $(5.27 \mathrm{eV}))$. As a result, the band bending occurs at the interface of $\mathrm{BiVO}_{4}$ and rGO, which favors the transfer of electrons from $\mathrm{BiVO}_{4}$ to rGO. ${ }^{54}$ In addition to this, electron transportation is also favored due to the presence of delocalized electrons on the $\pi-\pi$ graphitic carbon network and the higher conductivity of rGO. ${ }^{55}$ In addition to these factors, it is also reported that the piezoelectric polarization field also provides further band bending. ${ }^{4,46}$ All these factors favor the migration of free electrons from $\mathrm{BiVO}_{4}$ to $\mathrm{rGO}$, and hence increase the lifetime of free charge carriers. Thus, these free charge carriers have more time to participate in the piezocatalytic surface reactions. During the piezocatalytic surface reactions, the free electrons on the rGO surface get captured by adsorbed oxygen $\left(\mathrm{O}_{2}\right)$ to generate superoxide radicals $\left({ }^{\bullet} \mathrm{O}_{2}{ }^{-}\right)$, while the holes in the $\mathrm{VB}$ of $\mathrm{BiVO}_{4}$ oxidize hydroxide ions $\left(\mathrm{OH}^{-}\right)$to generate hydroxyl $\left({ }^{\bullet} \mathrm{OH}\right)$ radicals. These generated ${ }^{\bullet} \mathrm{O}_{2}{ }^{-}$and ${ }^{\bullet} \mathrm{OH}$ radicals degrade $\mathrm{MB}$ dye into harmless degradation products such as $\mathrm{CO}_{2}$ and $\mathrm{H}_{2} \mathrm{O} .^{56,57}$ The piezocatalytic reactions can be summarized in the form of eqn (2)-(6) provided as follows:

$$
\begin{aligned}
& \mathrm{BiVO}_{4}+\text { sonication } \rightarrow \mathrm{BiVO}_{4}\left(\mathrm{e}^{-}+\mathrm{h}^{+}\right) \\
& \mathrm{BiVO}_{4}\left(\mathrm{e}^{-}\right)+\mathrm{rGO} \rightarrow \mathrm{BiVO}_{4}+\mathrm{rGO}\left(\mathrm{e}^{-}\right)
\end{aligned}
$$




$$
\begin{gathered}
\mathrm{rGO}\left(\mathrm{e}^{-}\right)+\mathrm{O}_{2} \rightarrow \cdot{ }^{\bullet} \mathrm{O}_{2}^{-} \\
\mathrm{BiVO}_{4}\left(\mathrm{~h}^{+}\right)+\mathrm{OH}^{-} \rightarrow{ }^{\bullet} \mathrm{OH} \\
\cdot \mathrm{OH} / \mathrm{O}_{2}{ }^{-}+\mathrm{MB} \text { dye } \rightarrow \text { degradation products }
\end{gathered}
$$

\section{Conclusions}

rGO-BiVO ${ }_{4}$ composites were synthesized for piezocatalytic MB dye degradation. The piezocatalytic dye degradation performance increased initially with increment in rGO content up to $2 \mathrm{wt} \%$ followed by a decrease with further increment in rGO content. The synthesized $2 \mathrm{wt} \%$ rGO-BiVO ${ }_{4}$ composite demonstrated superior piezocatalytic performance to the other $\mathrm{BiVO}_{4}$ composites, which can be ascribed to the presence of interfacial coupling established between $\mathrm{rGO}$ and $\mathrm{BiVO}_{4}$, which causes an enhanced charge separation ability and enhanced carrier mobi-

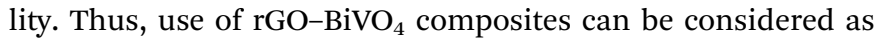
a novel method to ensure effective dye degradation through piezocatalysis.

\section{Conflicts of interest}

The authors declare not having any conflicts of interest.

\section{Acknowledgements}

RV thanks CSIR, New Delhi, for financial support under the EMR Scheme (22/0791/19/EMR-II).

\section{References}

1 M. Fakhrul Ridhwan Samsudin, S. Sufian, R. Bashiri, N. Muti Mohamed, L. Tau Siang and R. Mahirah Ramli, Optimization of photodegradation of methylene blue over modified $\mathrm{TiO}_{2} / \mathrm{BiVO}_{4}$ photocatalysts: Effects of total $\mathrm{TiO}_{2}$ loading and different type of co-catalyst, Mater. Today Proc., 2018, 5, 21710-21717, DOI: 10.1016/j.matpr.2018.07.023.

2 D. S. L. Balan and R. T. R. Monteiro, Decolourisation of textile dyes indigo dye by lignolytic fungi, J. Biotechnol., 2001, 89, 141-145.

3 H. Lei, H. Zhang, Y. Zou, X. Dong, Y. Jia and F. Wang, Synergetic photocatalysis/piezocatalysis of bismuth oxybromide for degradation of organic pollutants, J. Alloys Compd., 2019, 809, 151840, DOI: 10.1016/j.jallcom.2019.151840.

4 C. Hu, H. Huang, F. Chen, Y. Zhang, H. Yu and T. Ma, Coupling Piezocatalysis and Photocatalysis in $\mathrm{Bi}_{4} \mathrm{NbO}_{8} \mathrm{X}$ (X = Cl, Br) Polar Single Crystals, Adv. Funct. Mater., 2020, 30, 1-10, DOI: 10.1002/adfm.201908168.

5 D. Liu, Y. Song, Z. Xin, G. Liu, C. Jin and F. Shan, Highpiezocatalytic performance of eco-friendly $\left(\mathrm{Bi}_{1 / 2} \mathrm{Na}_{1 / 2}\right) \mathrm{TiO}_{3}$ based nanofibers by electrospinning, Nano Energy, 2019, 65, 104024, DOI: 10.1016/j.nanoen.2019.104024.

6 E. Lin, J. Wu, N. Qin, B. Yuan and D. Bao, Silver modified barium titanate as a highly efficient piezocatalyst, Catal. Sci. Technol., 2018, 8, 4788-4796, DOI: 10.1039/c8cy01127c.
7 B. Yuan, J. Wu, N. Qin, E. Lin, Z. Kang and D. Bao, Smdoped $\mathrm{Pb}\left(\mathrm{Mg}_{1 / 3} \mathrm{Nb}_{2 / 3}\right) \mathrm{O}_{3}-x \mathrm{PbTiO}_{3}$ piezocatalyst: Exploring the relationship between piezoelectric property and piezocatalytic activity, Appl. Mater. Today, 2019, 17, 183-192, DOI: 10.1016/j.apmt.2019.07.015.

8 Y. Zhang, X. Huang and J. Yeom, A Floatable Piezo-Photocatalytic Platform Based on Semi-Embedded ZnO Nanowire Array for High-Performance Water Decontamination, NanoMicro Lett., 2019, 11, 1-14, DOI: 10.1007/s40820-019-0241-9.

9 J. M. Wu, W. E. Chang, Y. T. Chang and C. K. Chang, PiezoCatalytic Effect on the Enhancement of the Ultra-High Degradation Activity in the Dark by Single- and Few-Layers $\mathrm{MoS}_{2}$ Nanoflowers, Adv. Mater., 2016, 28, 3718-3725, DOI: 10.1002/adma.201505785.

10 S. Singh and N. Khare, Coupling of piezoelectric, semiconducting and photoexcitation properties in $\mathrm{NaNbO}_{3}$ nanostructures for controlling electrical transport: Realizing an efficient piezo-photoanode and piezo-photocatalyst, Nano Energy, 2017, 38, 335-341, DOI: 10.1016/j.nanoen.2017.05.029.

11 A. Yourdkhani and G. Caruntu, Characterization of the microstructural and piezoelectric properties of $\mathrm{PbTiO}_{3}$ thin films synthesized by liquid-phase deposition, J. Phys. Chem. $C$, 2011, 115, 14797-14805, DOI: 10.1021/jp202127t.

12 M. Pan, C. Zhang, J. Wang, J. W. Chew, G. Gao and B. Pan, Multifunctional Piezoelectric Heterostructure of $\mathrm{BaTiO}_{3} @$ Graphene: Decomplexation of Cu-EDTA and Recovery of $\mathrm{Cu}$, Environ. Sci. Technol., 2019, 53, 8342-8351, DOI: 10.1021/ acs.est.9b02355.

13 X. Zhou, F. Yan, S. Wu, B. Shen, H. Zeng and J. Zhai, Remarkable Piezophoto Coupling Catalysis Behavior of BiOX/BaTiO 3 (X = Cl, Br, $\mathrm{Cl}_{0.166} \mathrm{Br}_{0.834}$ ) Piezoelectric Composites, Small, 2020, 16, 1-15, DOI: 10.1002/smll.202001573.

14 R. Su, H. A. Hsain, M. Wu, D. Zhang, X. Hu, Z. Wang, X. Wang, F. tang Li, X. Chen, L. Zhu, Y. Yang, Y. Yang, X. Lou and S. J. Pennycook, Nano-Ferroelectric for High Efficiency Overall Water Splitting under Ultrasonic Vibration, Angew. Chem., Int. Ed., 2019, 58, 15076-15081, DOI: 10.1002/anie.201907695.

15 J. Wang, T. Hou, F. Cao, M. Li and L. Lv, Harvesting the Vibration Energy with $\mathrm{BaTiO}_{3} @ G r a p h e n e$ for the Piezocatalytic Degradation of Methylene Blue, J. Environ. Sci. Eng. Technol., 2020, 8, 84-91, DOI: 10.12974/2311-8741.2020.08.8.

16 X. Zhou, B. Shen, J. Zhai and N. Hedin, Reactive Oxygenated Species Generated on Iodide-Doped $\mathrm{BiVO}_{4} / \mathrm{BaTiO}_{3}$ Heterostructures with $\mathrm{Ag} / \mathrm{Cu}$ Nanoparticles by Coupled Piezophototronic Effect and Plasmonic Excitation, Adv. Funct. Mater., 2021, 2009594.

17 X. Zhou, S. Wu, C. Li, F. Yan, H. Bai, B. Shen, H. Zeng and J. Zhai, Piezophototronic effect in enhancing charge carrier separation and transfer in $\mathrm{ZnO} / \mathrm{BaTiO}_{3}$ heterostructures for high-efficiency catalytic oxidation, Nano Energy, 2019, 66, 104127, DOI: 10.1016/j.nanoen.2019.104127.

18 P. Zhu, Y. Chen and J. Shi, Piezocatalytic Tumor Therapy by Ultrasound-Triggered and $\mathrm{BaTiO}_{3}$-Mediated Piezoelectricity, Adv. Mater., 2020, 32, 1-8, DOI: 10.1002/adma.202001976.

19 T. Wang, C. Li, J. Ji, Y. Wei, P. Zhang, S. Wang, X. Fan and J. Gong, Reduced graphene oxide $(\mathrm{rGO}) / \mathrm{BiVO}_{4}$ composites 
with maximized interfacial coupling for visible light photocatalysis, ACS Sustainable Chem. Eng., 2014, 2, 2253-2258, DOI: $10.1021 / \mathrm{sc5} 004665$.

20 J. K. Cooper, S. Gul, F. M. Toma, L. Chen, P. A. Glans, J. Guo, J. W. Ager, J. Yano and I. D. Sharp, Electronic structure of monoclinic $\mathrm{BiVO}_{4}$, Chem. Mater., 2014, 26, 5365-5373, DOI: $10.1021 / \mathrm{cm} 5025074$.

21 M. B. Tahir, T. Iqbal, H. Kiran and A. Hasan, Insighting role of reduced graphene oxide in $\mathrm{BiVO}_{4}$ nanoparticles for improved photocatalytic hydrogen evolution and dyes degradation, Int. J. Energy Res., 2019, 43, 2410-2417, DOI: 10.1002/er.4443.

22 R. Munprom, P. A. Salvador and G. S. Rohrer, Polar domains at the surface of centrosymmetric $\mathrm{BiVO}_{4}$, Chem. Mater., 2014, 26, 2774-2776, DOI: 10.1021/cm501087j.

23 G. Singh, M. Kumar and R. Vaish, Promising multicatalytic and adsorption capabilities in $\mathrm{V}_{2} \mathrm{O}_{5} / \mathrm{BiVO}_{4}$ composite pellets for water-cleaning application, Surf. Interfaces, 2021, 23, 100924, DOI: 10.1016/j.surfin.2021.100924.

24 N. Li, G. Liu, C. Zhen, F. Li, L. Zhang and H. M. Cheng, Battery performance and photocatalytic activity of mesoporous anatase $\mathrm{TiO}_{2}$ nanospheres/graphene composites by template-free self-assembly, Adv. Funct. Mater., 2011, 21, 1717-1722, DOI: 10.1002/adfm.201002295.

25 T. Soltani, A. Tayyebi and B. K. Lee, Photolysis and photocatalysis of tetracycline by sonochemically heterojunctioned $\mathrm{BiVO}_{4} /$ reduced graphene oxide under visible-light irradiation, J. Environ. Manage., 2019, 232, 713-721, DOI: 10.1016/ j.jenvman.2018.11.133.

26 S. Xiong, T. Wu, Z. Fan, D. Zhao, M. Du and X. Xu, Preparation of a leaf-like $\mathrm{BiVO}_{4}$-reduced graphene oxide composite and its photocatalytic activity, J. Nanomater., 2017, 2017, 3475248, DOI: 10.1155/2017/3475248.

27 J. G. Nam, E. S. Lee, W. C. Jung, Y. J. Park, B. H. Sohn, S. C. Park, J. S. Kim and J. Y. Bae, Photovoltaic enhancement of dye-sensitized solar cell prepared from $\left[\mathrm{TiO}_{2} /\right.$ ethyl cellulose/terpineol] paste employing TRITON $^{\mathrm{TM}}$ X-based surfactant with carboxylic acid group in the oxyethylene chain end, Mater. Chem. Phys., 2009, 116, 46-51, DOI: 10.1016/j.matchemphys.2009.02.037.

28 S. Tokunaga, H. Kato and A. Kudo, Selective preparation of monoclinic and tetragonal $\mathrm{BiVO}_{4}$ with scheelite structure and their photocatalytic properties, Chem. Mater., 2001, 13, 4624-4628, DOI: 10.1021/cm0103390.

29 B. Xu, X. Guan, L. Y. Zhang, X. Liu, Z. Jiao, X. Liu, X. Hu and X. S. Zhao, A simple route to preparing $\gamma-\mathrm{Fe}_{2} \mathrm{O}_{3} / \mathrm{RGO}$ composite electrode materials for lithium ion batteries, J. Mater. Chem. A, 2018, 6, 4048-4054, DOI: 10.1039/c7ta10052c.

30 B. $\mathrm{Wu}, \mathrm{G}$. Zheng and X. Chen, Effect of graphene on the thermophysical properties of melamine-urea-formaldehyde/ N-hexadecane microcapsules, RSC Adv., 2015, 5, 74024-74031, DOI: $10.1039 / \mathrm{c} 5 \mathrm{ra12566a.}$

31 L. M. Rivera, A. F. Betancur, D. G. Zarate, D. T. Torres, L. M. Hoyos and A. G. Garcia, Reduction and simultaneous doping of graphene oxide to repel LDL in treatment of atherosclerosis disease, 2019, arXiv:1902.01850.
32 A. Tayyebi, T. Soltani, H. Hong and B. K. Lee, Improved photocatalytic and photoelectrochemical performance of monoclinic bismuth vanadate by surface defect states $\left(\mathrm{Bi}_{1-\mathrm{x}} \mathrm{VO}_{4}\right)$, J. Colloid Interface Sci., 2018, 514, 565-575, DOI: 10.1016/j.jcis.2017.12.067.

$33 \mathrm{~S}$. Nikam and S. Joshi, Irreversible phase transition in $\mathrm{BiVO}_{4}$ nanostructures synthesized by a polyol method and enhancement in photo degradation of methylene blue, RSC Adv. , 2016, 6, 107463-107474, DOI: 10.1039/c6ra14700c.

34 A. R. Nanakkal and L. K. Alexander, Graphene/BiVO $4 / \mathrm{TiO}_{2}$ nanocomposite: tuning band gap energies for superior photocatalytic activity under visible light, J. Mater. Sci., 2017, 52, 7997-8006, DOI: 10.1007/s10853-017-1002-0.

35 S. Stankovich, D. A. Dikin, R. D. Piner, K. A. Kohlhaas, A. Kleinhammes, Y. Jia, Y. Wu, S. B. T. Nguyen and R. S. Ruoff, Synthesis of graphene-based nanosheets via chemical reduction of exfoliated graphite oxide, Carbon, 2007, 45, 1558-1565, DOI: 10.1016/j.carbon.2007.02.034.

36 D. Yao, C. Dong, Q. Bing, Y. Liu, F. Qu, M. Yang, B. Liu, B. Yang and H. Zhang, Oxygen-Defective Ultrathin $\mathrm{BiVO}_{4}$ Nanosheets for Enhanced Gas Sensing, ACS Appl. Mater. Interfaces, 2019, 11, 23495-23502, DOI: 10.1021/acsami.9b05626.

37 N. Moslemzadeh, G. Beamson, P. Tsakiropoulos, J. F. Watts, S. R. Haines and P. Weightman, The 1s XPS spectra of the 3d transition metals from scandium to cobalt, $J$. Electron Spectrosc. Relat. Phenom., 2006, 152, 129-133, DOI: 10.1016/ j.elspec.2006.03.011.

38 H. Tian, H. Wu, Y. Fang, R. Li and Y. Huang, Hydrothermal synthesis of $\mathrm{m}-\mathrm{BiVO}_{4} / \mathrm{t}-\mathrm{BiVO}_{4}$ heterostructure for organic pollutants degradation: Insight into the photocatalytic mechanism of exposed facets from crystalline phase controlling, J. Hazard. Mater., 2020, 399, 123159, DOI: 10.1016/ j.jhazmat.2020.123159.

39 N. Daelman, F. S. Hegner, M. Rellán-Piñeiro, M. CapdevilaCortada, R. García-Muelas and N. López, Quasi-degenerate states and their dynamics in oxygen deficient reducible metal oxides, J. Chem. Phys., 2020, 152(5), 050901, DOI: 10.1063/1.5138484.

40 G. Tezcanli-Güyer and N. H. Ince, Individual and combined effects of ultrasound, ozone and UV irradiation: A case study with textile dyes, Ultrasonics, 2004, 42, 603-609, DOI: 10.1016/j.ultras.2004.01.096.

41 N. Ghows and M. H. Entezari, Kinetic investigation on sonodegradation of Reactive Black 5 with core-shell nanocrystal, Ultrason. Sonochem., 2013, 20, 386-394, DOI: 10.1016/ j.ultsonch.2012.06.013.

42 J. H. Shah, M. Fiaz, M. Athar, J. Ali, M. Rubab, R. Mehmood, S. U. U. Jamil and R. Djellabi, Facile synthesis of N/B-double-doped $\mathrm{Mn}_{2} \mathrm{O}_{3}$ and $\mathrm{WO}_{3}$ nanoparticles for dye degradation under visible light, Environ. Technol., 2020, 41, 2372-2381, DOI: 10.1080/09593330.2019.1567604.

43 U. Alam, A. Khan, D. Ali, D. Bahnemann and M. Muneer, Comparative photocatalytic activity of sol-gel derived rare earth metal (La, Nd, Sm and Dy)-doped ZnO photocatalysts for degradation of dyes, RSC Adv., 2018, 8, 17582-17594, DOI: $10.1039 / \mathrm{c} 8 \mathrm{ra} 01638 \mathrm{k}$. 
44 M. Ismail, Z. Wu, L. Zhang, J. Ma, Y. Jia, Y. Hu and Y. Wang, High-efficient synergy of piezocatalysis and photocatalysis in bismuth oxychloride nanomaterial for dye decomposition, Chemosphere, 2019, 228, 212-218, DOI: 10.1016/ j.chemosphere.2019.04.121.

45 X. Wang, L. Zhi and K. Müllen, Transparent, conductive graphene electrodes for dye-sensitized solar cells, Nano Lett., 2008, 8, 323-327, DOI: 10.1021/nl072838r.

46 Z. Zhao, L. Wei, S. Li, L. Zhu, Y. Su, Y. Liu, Y. Bu, Y. Lin, W. Liu and Z. Zhang, Exclusive enhancement of catalytic activity in $\mathrm{Bi}_{0.5} \mathrm{Na}_{0.5} \mathrm{TiO}_{3}$ nanostructures: new insights into the design of efficient piezocatalysts and piezo-photocatalysts, J. Mater. Chem. A, 2020, 8, 16238-16245, DOI: 10.1039/c9ta14007g.

47 C. $\mathrm{Hu}, \mathrm{S} . \mathrm{Tu}, \mathrm{N}$. Tian, T. Ma, Y. Zhang and H. Huang, External fields enhanced photocatalysis, Angew. Chem., Int. Ed., 2020, DOI: 10.1002/anie.202009518.

48 S. Tu, Y. Guo, Y. Zhang, C. Hu, T. Zhang, T. Ma and H. Huang, Piezocatalysis and Piezo-Photocatalysis: Catalysts Classification and Modification Strategy, Reaction Mechanism, and Practical Application, Adv. Funct. Mater., 2020, 30, 1-31, DOI: 10.1002/adfm.202005158.

49 F. Chen, H. Huang, L. Guo, Y. Zhang and T. Ma, The Role of Polarization in Photocatalysis, Angew. Chem., Int. Ed., 2019, 58, 10061-10073, DOI: 10.1002/anie.201901361.

$50 \mathrm{~J} . \mathrm{Wu}, \mathrm{N}$. Qin and D. Bao, Effective enhancement of piezocatalytic activity of $\mathrm{BaTiO}_{3}$ nanowires under ultrasonic vibration, Nano Energy, 2018, 45, 44-51, DOI: 10.1016/ j.nanoen.2017.12.034.

51 Y. Feng, L. Ling, Y. Wang, Z. Xu, F. Cao, H. Li and Z. Bian, Engineering spherical lead zirconate titanate to explore the essence of piezo-catalysis, Nano Energy, 2017, 40, 481-486, DOI: 10.1016/j.nanoen.2017.08.058.

52 J. W. Larsen, M. Freund, K. Y. Kim, M. Sidovar and J. L. Stuart, 00/03269 Mechanism of the carbon catalysed reduction of nitrobenzene by hydrazine, Fuel Energy Abstr., 2000, 41, 368, DOI: 10.1016/s0140-6701(00)94344-3.

53 Y. A. J. Al-Hamadani, G. Lee, S. Kim, C. M. Park, M. Jang, N. Her, J. Han, D. H. Kim and Y. Yoon, Sonocatalytic degradation of carbamazepine and diclofenac in the presence of graphene oxides in aqueous solution, Elsevier Ltd, 2018, DOI: 10.1016/j.chemosphere.2018.04.129.

54 S. Bai, L. Sun, J. Sun, J. Han, K. Zhang, Q. Li, R. Luo, D. Li and A. Chen, Pine dendritic bismuth vanadate loaded on reduced graphene oxide for detection of low concentration triethylamine, J. Colloid Interface Sci., 2021, 587, 183-191, DOI: $10.1016 /$ j.jcis.2020.10.113.

55 J. Prakash, U. Prasad, R. Alexander, J. Bahadur, K. Dasgupta and A. N. M. Kannan, Photoelectrochemical Solar Water Splitting: The Role of the Carbon Nanomaterials in Bismuth Vanadate Composite Photoanodes toward Efficient Charge Separation and Transport, Langmuir, 2019, 35, 14492-14504, DOI: 10.1021/acs.langmuir.9b02782.

56 A. Biswas, S. Saha and N. R. Jana, $\mathrm{ZnSnO}_{3}$-hBN nanocompositebased piezocatalyst: ultrasound assisted reactive oxygen species generation for degradation of organic pollutants, New J. Chem., 2020, 44, 9278-9287, DOI: 10.1039/d0nj01026j.

57 Y. Sun, B. Qu, Q. Liu, S. Gao, Z. Yan, W. Yan, B. Pan, S. Wei and Y. Xie, Highly efficient visible-light-driven photocatalytic activities in synthetic ordered monoclinic $\mathrm{BiVO}_{4}$ quantum tubes-graphene nanocomposites, Nanoscale, 2012, 4, 3761-3767, DOI: 10.1039/c2nr30371j. 